\title{
Cell-free RNA studies in cancer based on T oligo-primed polymerase chain reaction (TOP-PCR) technology
}

\author{
K.-P. Chiu ${ }^{1}$, N.V. Gubanova ${ }^{2}$, A.V. Tsukanov², S.S. Kovalev²,3, Y.L. Orlov²,4* \\ ${ }^{1}$ Genomics Research Center, Academia Sinica, Taipei, Taiwan \\ ${ }^{2}$ Institute of Cytology and Genetics SB RAS, Novosibirsk, Russia \\ ${ }^{3}$ Novosibirsk State Medical University, Novosibirsk, Russia \\ ${ }^{4}$ Novosibirsk State University, Novosibirsk, Russia \\ *e-mail:orlov@bionet.nsc.ru
}

Key words: sequencing technology, transcriptome, glioblastoma, splicing, cancer

Motivation and Aim: We suggest joint research project devoted to the development of cell-free RNA (cfRNA) analysis technology using TOP-PCR. The project relies on the expertise of Taiwanese group in TOP-PCR technology development and the experience of Russian science team in gene expression and transcriptomics data analysis and network reconstruction. The TOP-PCR technology [1] will be applied for analysis of biomarkers in cancer and other diseases. Body fluid nucleic acid sequencing is a powerful noninvasive approach for the diagnosis of genetic defects, infectious agents and diseases. The success relies on the quantity and quality of the DNA samples.

Methods and Algorithms: Numerous clinical samples are either at low quantity or of poor quality due to various reasons. To overcome these problems, Taiwanese group have developed T oligo-primed polymerase chain reaction (TOP-PCR) for full-length nonselective amplification of minute quantity of DNA fragments. TOP-PCR adopts homogeneous "half adaptor" (HA), generated by annealing P oligo (carrying a phosphate group at the $5^{\prime}$ end) and T oligo (carrying a T-tail at the $3^{\prime}$ end), for efficient ligation to target DNA and subsequent PCR amplification primed by the T oligo alone.

Results: The preliminary results also showed that TOP-PCR is a superior method for detecting apoptosis and outperforms the method adopted by Illumina for DNA amplification.

Conclusion: We will analyze cancer mutations in cell-free DNA and develop methods of gene network reconstruction and computer models of non-coding RNA interactions with focus on cell-free RNA. Gene expression analysis will allow find biomarkers associated with cancer, especially for glioblastoma [2]. The group will use text mining of science literature and associate gene network reconstruction methods to find related genes and RNA targets. Associative gene network reconstruction will be based on ANDVisio and ANDSystem tools; gene network modeling will be based on TRRD and GeneNet databases developed at ICG SB RAS. The project has fundamental role for biomarkers studies and could be applied for wide range of biotechnology problems.

Acknowledgements: The work was supported by RFBR No. 18-29-09105 and ICG SB RAS budget project No. 0324-2018-0019.

\section{References}

1. Nai Y.-S. et al. (2017) T Oligo-Primed Polymerase Chain Reaction (TOP-PCR): A Robust Method for the Amplification of Minute DNA Fragments in Body Fluids. Scientific Reports. 7:40767.

2. Babenko V.N. et al. (2017) Computer Analysis of Glioma Transcriptome Profiling: Alternative Splicing Events. Journal of Integrative Bioinformatics. 14(3). pii: /j/jib.2017.14.issue-3/jib-2017-0022/jib-20170022.xml. 